\title{
Intraductal papillary neoplasm of the bile duct: two case reports and review of the literature
}

\author{
Vishal Kaila*1, Siddharth B Javia ${ }^{2}$, Nirav Thosani ${ }^{2}$, Isaac Raijman ${ }^{3}$ \\ ${ }^{1}$ Department of Medicine, Texas Health Dallas, Dallas, Texas, United States \\ ${ }^{2}$ Department of Internal Medicine, Division of Gastroenterology, Hepatology \& Nutrition; University of Texas Medical School at \\ Houston, Texas, United States \\ ${ }^{3}$ Department of Gastroenterology, Baylor College of Medicine, Houston, Texas, United States
}

Received: March 4, 2019

DOI: $10.5430 /$ crim.v6n2p25
Accepted: May 19, 2019

Online Published: June 3, 2019

\begin{abstract}
Background: Intraductal Papillary Neoplasm of the Bile Duct (IPNB) is a rare disease that is characterized by papillary lesions in the intra and extrahepatic biliary tree. Traditional imaging studies may show biliary dilation and/or filling defects. Endoscopic retrograde cholangiopancreatography can demonstrate diffuse bile duct dilatation as well as amorphous filling defects, however visualization of the biliary system can be limited by obstruction by the papillary lesions or mucin. Digital single operator cholangioscopy can be used to aid in diagnosis.

Case Presentation: We report two elderly Caucasian males that presented with fatigue and obstructive jaundice. In both cases, the diagnosis of IPNB of was made. In one case, digital single operator cholangioscopy was used successfully to make the diagnosis.

Conclusions: We present two cases of IPNB as well as a review of the clinical characteristics of IPNB and the utility of digital single operator cholangioscopy to aid in diagnosis. In IPNB, timely diagnosis is paramount. Traditional imaging studies can be inadequate, digital single operator cholangioscopy allows for quick diagnosis and, if indicated, further intervention.
\end{abstract}

Key Words: Intraductal Papillary Neoplasm of the Bile Duct, IPNB, Biliary Papillomatosis, Cholangioscopy, Spyglass

\section{INTRODUCTION}

Intraductal Papillary Neoplasm of the Bile Duct (IPNB) is a rare condition that is characterized by numerous papillary lesions in the intra and extrahepatic biliary tree. Patients present with right upper quadrant pain and symptoms associated with obstructive jaundice. Due to the rarity of IPNB as well as the multitude of other diagnosis associated with obstructive jaundice, final diagnosis can be difficult. In addition, the papillary lesions have shown to have a high degree of malignant transformation, thus necessitating the need for rapid diagnosis. Here we present 2 cases of a patients diagnosed with IPNB after presenting with obstructive jaundice.
In one case, the patient was diagnosed with Endoscopic retrograde cholangiopancreatography (ERCP) and the other case was diagnosed with digital single operator cholangioscopy (DSOC).

\section{Case presentations}

\subsection{Case 1}

A 68-year-old male with past medical history significant for follicular lymphoma in remission presented from an outside facility for management of obstructive jaundice as well as cholangitis. Patient reported decreased appetite, night sweats in addition to a 20-pound weight loss over the past 20 days. His admission labs were significant for: total

\footnotetext{
*Correspondence: Vishal Kaila; Email: vishalkaila@yahoo.com; Address: Presbyterian Hospital of Dallas 8200 Walnut Hill Lane, Dallas, TX 75231, United States.
} 
bilirubin-11.2 mg/dl (0.2-1.2 mg/dl), alkaline phosphatase$276 \mathrm{U} / \mathrm{L}(53-128 \mathrm{U} / \mathrm{L})$ and white blood cell count- $13.5 \mathrm{k} / \mathrm{ul}$ (3.98-9.57 k/ul). Coronal CT of the Abdomen (see Figure 1) showed multiple intraductal masses in intrahepatic bile ducts bilaterally (red arrows) and common hepatic duct (CHD, yellow arrow) with upstream intrahepatic biliary dilation. Coronal 2D MRCP (see Figure 2) of the liver showed filling defects corresponding to multiple intraductal masses (arrows) in intrahepatic bile ducts bilaterally and common hepatic duct with upstream intrahepatic biliary dilation. Endoscopic ultrasound(see Figure 3) showed large hyper-echoic lesions in the left lobe of the liver with adjacent intrahepatic cystic ductal dilatation or cystic mass with internal tumor. Fine-need-aspiration cytology was obtained from the liver lesion. ERCP (see Figure 4) showed Bismuth IV biliary stricture involving CHD and both right and left main hepatic ducts with upstream biliary dilatation. Biopsies were obtained from CHD stricture using pediatric forceps. Bilateral plastic biliary stents were placed. Biopsy of CHD stricture showed biliary type epithelium with extensive low and high-grade dysplasia. Biliary brushing showed malignant cells consistent with adenocarcinoma. Cytology of liver mass showed malignant cells of biliary or pancreatic origin. Patient subsequently underwent left hepatic lobectomy and hepatico-jejunostomy. Surgical pathology showed minimally invasive cholangiocarcinoma arising multifocally in an IPNB with low and high-grade dysplasia (see Figures 5, 6). No lymphovascular or perineural invasion was seen. Surgical margins were negative for carcinoma or high-grade dysplasia. Patient underwent adjuvant chemotherapy and radiation. Positron emission tomography obtained 4 months after the surgery did not show evidence of disease.

\subsection{Case 2}

A 52-year male was referred to clinic after experiencing right upper quadrant pain, itching, fatigue and intermittent confusion for several weeks. He endorsed previous episodes of jaundice that resolved spontaneously and has family history significant for his grandmother and uncle being previously diagnosed with pancreatic cancer. Vital signs were within normal limits and physical exam was notable for right upper quadrant pain. Laboratory studies showed an alanine aminotransferase of $539 \mathrm{U} / \mathrm{L}$ (0-55 U/L), aspartate aminotransferase of 307 (5-34 U/L), Alkaline Phosphatase of 1,162 $\mathrm{U} / \mathrm{L}$ (53-128 U/L) and Total bilirubin of $2.9 \mathrm{mg} / \mathrm{dl}(0.2-1.2$ $\mathrm{mg} / \mathrm{dl})$. CT scan showed intrahepatic biliary dilatation secondary to obstruction of the central common bile duct as well as an ill-defined mass in the right lobe of the liver (see Figure 1). DSOC was used to directly visualize the biliary tree in this patient, and the diagnosis of IPNB was made (see Figure 2, 3).

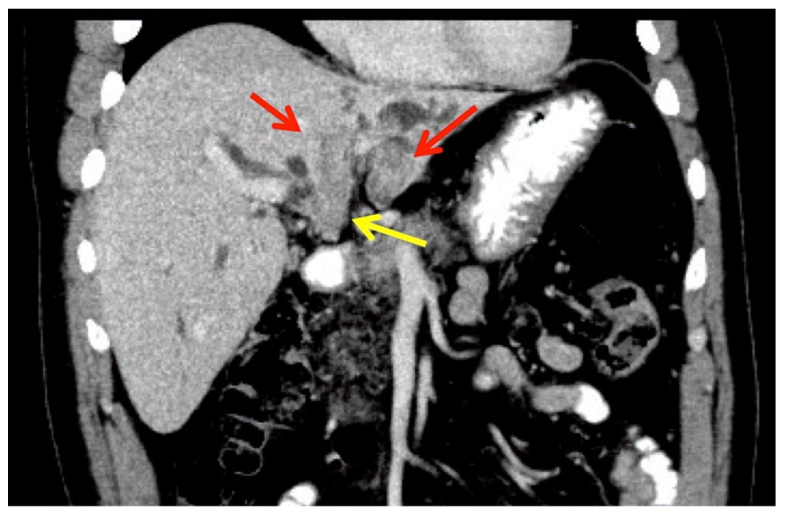

Figure 1. Coronal CT of the abdomen, multiple intraductal masses in intrahepatic bile ducts bilaterally (red arrows) and common hepatic duct (yellow arrow) with upstream intrahepatic biliary dilation

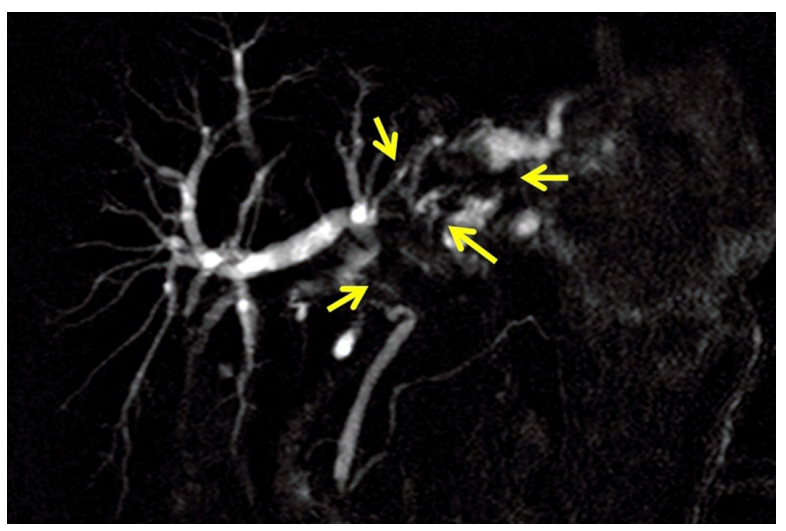

Figure 2. Coronal 2D MRCP of liver, Filling defects corresponding to multiple intraductal masses (arrows) in intrahepatic bile ducts bilaterally and common hepatic duct with upstream intrahepatic biliary dilation

\section{Discussion}

Although IPNB has been characterized in the literature, with the first case being described in $1894,{ }^{[1]}$ it is a very rare disease process defined as an exophytic biliary epithelial tumor with extensive intraductal papillary lesions. It is isolated to the extrahepatic ducts in $58 \%$ of cases with $33 \%$ of cases involving both the extra and intrahepatic ducts and the remaining 9\% affecting only the intrahepatic ducts. ${ }^{[2]}$ Microscopically, cells within IPNB lesions exhibit a spectrum of atypia ranging from no atypia to marked atypia and can also be associated with invasive carcinoma. ${ }^{[3]}$ Thus, IPNB is considered a premalignant lesion to cholangiocarcinoma, similar to intraductal papillary mucinous neoplasm (IPMN) of the pancreas. ${ }^{[3]}$ These lesions can cause biliary obstructions and, thus, patients commonly present with a combination of right upper quadrant pain with obstructive jaundice as well as cholangitis. However, a portion of patients, 5\%-29\%, are asymptomatic. ${ }^{[4-8]}$ 


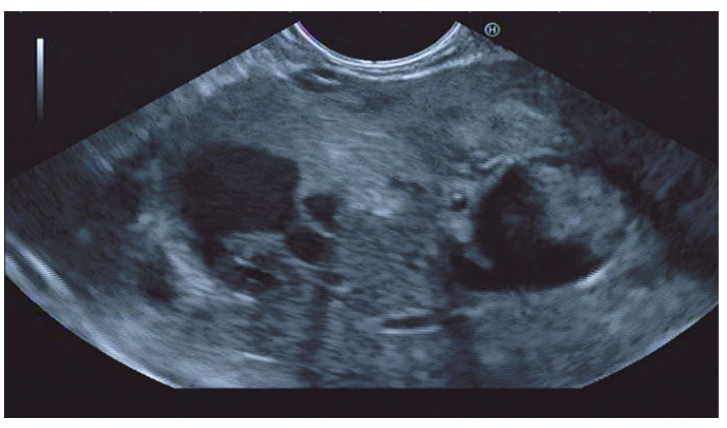

Figure 3. Endoscopic Ultrasound, large hyper-echoic lesions in the left lobe of the liver with adjacent intrahepatic cystic ductal dilatation or cystic mass with internal tumor

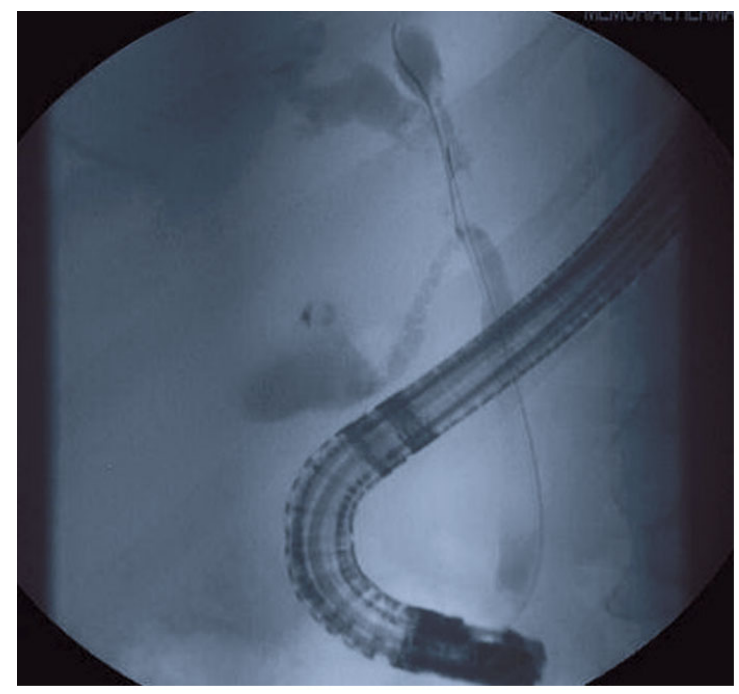

Figure 4. ERCP, stricture involving CHD and both right and left main hepatic ducts with upstream biliary dilatation

The incidence of IPNB is highest in the Far Eastern Countries because the risk factors for IPNB, hepatolithiasis and clonorchiasis, are endemic. ${ }^{[3]}$ IPNB affects males more frequently with a ratio of 2:1 and usually affects men in their sixth to seventh decade of life. ${ }^{[9,10]}$ The pathogenesis of IPNB is unclear, however the consequences of processes such as recurrent choledocholithiasis, pyogenic cholangitis or congenital cysts, could play a role in chronic biliary injury that could then later cause the proliferation of papillary cell in the biliary tree. ${ }^{[9,11,12]}$ IPNB is now known to have high malignant potential, with one study showing that 48 out of 58 patients (83\%) diagnosed with IPNB had pathology showing either adenocarcinoma or mucinous carcinoma. ${ }^{[12]}$

Radiologic diagnosis of IPNB is difficult due to the rarity of the disease as well as other confounding factors. Traditional cross-sectional imaging studies such as Ultrasonography (US), Computed Tomography (CT) and Magnetic Resonance Imaging (MRI) may show biliary dilation and/or filling defects. MRI studies of IPNB will show hypointense masses involving the biliary tree on $\mathrm{T} 1$ weighted images and hyperintense lesions on T2 weighted images. ${ }^{[13]}$ US, CT, and MRI will not show the presence or lack thereof mucin. Duodenoscopy can reveal a dilated papillary orifice with mucinous drainage and ERCP can demonstrate diffuse bile duct dilatation as well as amorphous filling defects, however complete visualization of the biliary system can be limited by obstruction by the papillary lesions or mucin produced by the lesions themselves. ${ }^{[14]}$ During ERCP, intraductal ultrasonography (IDUS) provides real time cross-sectional images of biliary tract to aide in locating as well as assessing the depth of biliary lesions. However, with IDUS biliary sludge can have the appearance of elevated tumors and, in cases of patients with acute cholangitis, it is difficult to assess the difference between superficial tumor spread and inflammatory biliary wall thickness. ${ }^{[14]}$

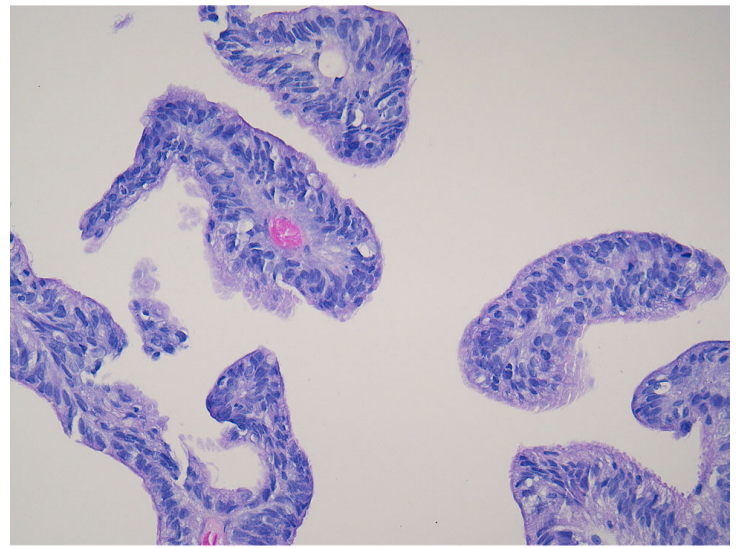

Figure 5. H\&E, $10 \times$ objective, Several papillary structures formed by epithelial cells with moderate nuclear pleomorphism, hyperchromasia, and loss of polarity with occasional mitotic figures consistent with high-grade dysplasia

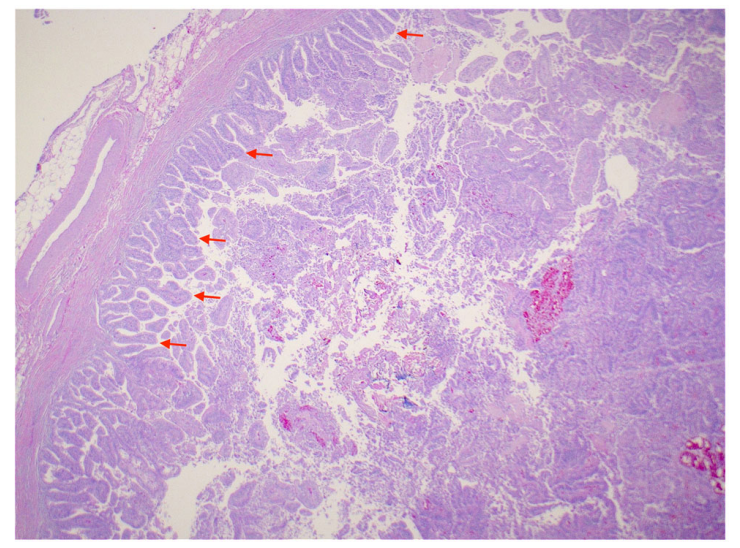

Figure 6. H\&E, $2 \times$ magnification, Low power magnification showing an intraductal growth formed by numerous papillary structures (red arrows) with low-grade and high-grade dysplasia 
Cholangioscopy performed either percutaneously transhepatic cholangioscopy (PTCS) or endoscopically allows for direct visualization of the bile duct lumen and epithelium. ${ }^{[15]}$ PTCS is associated with serious complications such as hemobilia and tumor seeding of the tract, however it is useful in lesions that cannot be reached by the peroral approach. ${ }^{[14]}$ Per-oral digital cholangioscopy (DSOC) is now the preferred method and the most commonly used cholangioscope is the Spyglasstm Direct Visualization System (Boston Scientific Corporation, USA). DSOC is a single operator cholangioscope which is attached to a therapeutic duodenoscope (4.2 $\mathrm{mm}$ channel) and, once the biliary tree is cannulated using standard methods, the visualization probe is inserted into the biliary ducts over a guidewire. ${ }^{[16]}$ DSOC has the capability to being introduced deep into the intra-hepatic biliary tree and can be used in any patient. Targeted biopsies can be obtained and the visual characteristics of the identified lesion (s) are sensitive and specific.

In contrast to IPMN of the pancreas, all cases of IPNB typically require intervention since patients will often have recurrent episodes of obstructive jaundice and cholangitis. For patients without distant metastasis, surgical resection is the

\section{REFERENCES}

[1] Caroli J. Papillomas and papillomatoses of the common bile duct. Rev Med Chir Mal Foie. 1959; 34: 191-230.

[2] Chung DJ, Lee SK, Ha HK, et al. Multiple biliary papillomatosis: comparison of MR cholangiography with endoscopic retrograde cholangiography. J Comput Assist Tomogr. 2002; 26: 968-974. PMid:12488745. https : //doi.org/10.1097/00004728-20021 1000-00020

[3] Ohtsuka M, Shimizu H, Kato A, et al. Intraductal papillary neoplasms of the bile duct. Int J Hepatol. 2014; 2014: 459091. PMid:24949206. https://doi.org/10.1155/2014/459091

[4] Ohtsuka M, Kimura F, Shimizu H, et al. Similarities and differences between intraductal papillary tumors of the bile duct with and without macroscopically visible mucin secretion. Am J Surg Pathol. 2011; 35: 512-521. PMid:21412069. https://doi .org/10.1097/PAS . Ob013e3182103f36

[5] Rocha FG, Lee H, Katabi N, et al. Intraductal papillary neoplasm of the bile duct: a biliary equivalent to intraductal papillary mucinous neoplasm of the pancreas? Hepatol. Baltim Md. 2012; 56: 1352-1360 PMid:22504729. https://doi .org/10.1002/hep. 25786

[6] Kim KM, Lee JK, Shin JU, et al. Clinicopathologic features of intraductal papillary neoplasm of the bile duct according to histologic subtype. Am J Gastroenterol. 2012; 107: 118-125. PMid:21946282. https://doi.org/10.1038/ajg.2011.316

[7] Choi SC, Lee JK, Jung JH, et al. The clinicopathological features of biliary intraductal papillary neoplasms according to the location of tumors. J Gastroenterol Hepatol. 2010; 25: 725-730. PMid:20492329. https://doi.org/10.1111/j.1440-1746.2009.06104.x

[8] Paik KY, Heo JS, Choi SH, et al. Intraductal papillary neoplasm of the bile ducts: the clinical features and surgical outcome of 25 cases. J Surg Oncol. 2008; 97: 508-512. PMid:18314868. https: //doi.org/10.1002/jso.20994 treatment of choice, usually involving pancreaticoduodenoectomy or major hepatatectomy with or without extrahepatic bile duct resection. ${ }^{[3]}$ For local disease that is confirmed preoperatively can undergo a limited resection to preserve anatomy. In extensive or multifocal disease, liver transplantation with pancreaticoduodenoectomy must be considered in cases without metastatic disease due to the high rates of recurrences after limited surgical resection.

In conclusion, IPNB is a rare premalignant biliary disorder characterized by obstructive jaundice and cholangitis. Timely diagnosis is paramount and traditional imaging studies do not provide sufficient diagnostic value. The use of DSOC and DPC facilitates early diagnosis and, if warranted, therapeutic intervention.

\section{ACKNOWLEDGEMENTS}

Each author was equally involved in study concept and design, acquisition of data, analysis and interpretation of data, drafting of the manuscript, and critical revision of the manuscript for important intellectual content.

\section{CONFlicts OF InTEREST Disclosure}

The authors declare no conflicts of interest.
[9] Yeung YP, AhChong K, Chung CK, et al. Biliary papillomatosis: report of seven cases and review of English literature. J Hepatobiliary Pancreat Surg. 2003; 10: 390-395. PMid:14598142. https: //doi.org/10.1007/s00534-002-0837-0

[10] Taguchi J, Yasunaga M, Kojiro M, et al. Intrahepatic and extrahepatic biliary papillomatosis. Arch Pathol Lab Med. 1993; 117: 944-947.

[11] Terada T, Nakanuma Y. Congenital biliary dilatation in autosomal dominant adult polycystic disease of the liver and kidneys. Arch. Pathol Lab Med. 1988; 112: 1113-1116.

[12] Lee PS, Auyeung KM, To KF, et al. Biliary papillomatosis complicating recurrent pyogenic cholangitis. Clin Radiol. 2001; 56: 591-593. PMid:11446759. https://doi.org/10.1053/crad.2000.0610

[13] Yoon HJ, Kim YK, Jang KT, et al. Intraductal papillary neoplasm of the bile ducts: description of MRI and added value of diffusion-weighted MRI. Abdom Imaging. 2013; 38: 10821090. PMid:23508835. https://doi.org/10.1007/s00261-0 13-9989-4

[14] Tsuyuguchi T, Sakai Y, Sugiyama H, et al. Endoscopic diagnosis of intraductal papillary mucinous neoplasm of the bile duct. J. Hepato-Biliary-Pancreat Sci. 2010; 17: 230-235. PMid:19669677. https://doi.org/10.1007/s00534-009-0153-z

[15] Parsi MA. Biliary papillomatosis: diagnosis with direct peroral cholangioscopy. Gastrointest Endosc. 2015; 81: 231-232. PMid:24929479. https://doi.org/10.1016/j.gie.2014.04. 040

[16] Chen YK, Pleskow DK. SpyGlass single-operator peroral cholangiopancreatoscopy system for the diagnosis and therapy of bile-duct disorders: a clinical feasibility study (with video). Gastrointest Endosc. 2007; 65: 832-841. PMid:17466202. https://doi.org/10 $.1016 /$ j.gie. 2007.01 .025 